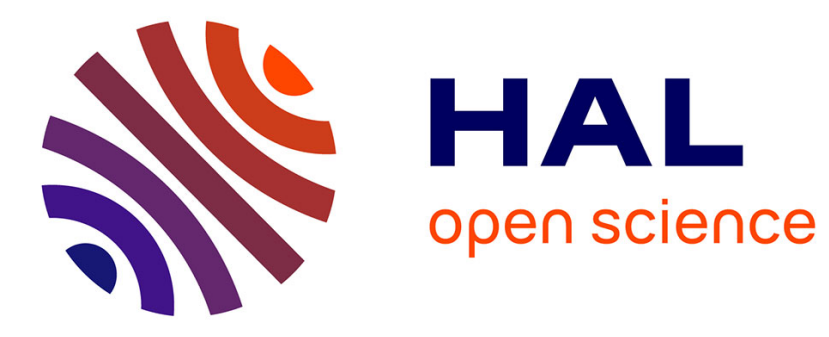

\title{
Admittance control for collaborative dual-arm manipulation
}

\author{
Sonny Tarbouriech, Benjamin Navarro, Philippe Fraisse, André Crosnier, \\ Andrea Cherubini, Damien Sallé
}

\section{- To cite this version:}

Sonny Tarbouriech, Benjamin Navarro, Philippe Fraisse, André Crosnier, Andrea Cherubini, et al.. Admittance control for collaborative dual-arm manipulation. ICAR 2019 - 19th International Conference on Advanced Robotics, Dec 2019, Belo Horizonte, Brazil. pp.198-204, 10.1109/ICAR46387.2019.8981624 . hal-02307959

\section{HAL Id: hal-02307959 \\ https://hal.science/hal-02307959}

Submitted on 8 Oct 2019

HAL is a multi-disciplinary open access archive for the deposit and dissemination of scientific research documents, whether they are published or not. The documents may come from teaching and research institutions in France or abroad, or from public or private research centers.
L'archive ouverte pluridisciplinaire HAL, est destinée au dépôt et à la diffusion de documents scientifiques de niveau recherche, publiés ou non, émanant des établissements d'enseignement et de recherche français ou étrangers, des laboratoires publics ou privés. 


\title{
Admittance control for collaborative dual-arm manipulation
}

\author{
Sonny Tarbouriech*† Benjamin Navarro* Philippe Fraisse* \\ André Crosnier* Andrea Cherubini* and Damien Sallé ${ }^{*}$
}

\begin{abstract}
Human-robot collaboration is an appealing solution to increase the flexibility of production lines. In this context, we propose a kinematic control strategy for dual-arm robotic platforms physically collaborating with human operators. Based on admittance control, our approach aims at improving the performance of object transportation tasks by acting on two levels: estimating and compensating gravity effects on one side, and considering human intention in the cooperative task space on the other. An experimental study using virtual reality reveals the effectiveness of our method in terms of reduced human energy expenditure.
\end{abstract}

\section{INTRODUCTION}

Letting several manipulator arms share the same workspace and operate cooperatively brings the potential of industrial robotics at a higher level [1]. As for humans, the combination of two arms makes the realisation of complex tasks possible [2], [3] and allows the manipulation of bigger and heavier objects than what would have been feasible with a unique arm [4].

Enabling physical human-robot collaboration has tremendous potential and is particularly attractive for industrial purposes [5]. Within the numerous applications, collaborative carrying has raised great interest for its ability to facilitate the transportation of bulky objects.

Many researchers have addressed the control problem of a robot system handling an object in cooperation with a human. Early work involving human-robot object comanipulation was done by Kosuge et al. [6]. They studied the general case of several humans collaborating with a multi-arm robotic system. Assuming a rigid grasp and no relative motion between the arms, the mechanical impedance of the commonly held object is controlled and the necessary impedance for each robotic arm is deduced. Taking a similar approach, the dual-arm mobile robot "MR helper" is able to collaborate with humans using wrench feedback from each arm's wrist [7]. The apparent impedance of the object is estimated to generate the command in the task space.

The need for robotic assistance is particularly desirable for moving cumbersome objects. In [8], the author proposes a method to facilitate the cooperative manipulation of long parts. By setting a virtual nonholonomic constraint at the tip of the robotic arm, the object's motion is restricted to a plane to prevent sideslip. Combining horizontal and vertical movements enables the 6 degrees of freedom (DOF) manipulation of the object in $3 \mathrm{D}$ space. The load sharing

\footnotetext{
* LIRMM, Université de Montpellier, CNRS, Montpellier, France. firstname. lastnamedlirmm.fr

$\dagger$ Tecnalia Research and Innovation, Industry and Transport Division.
}

during co-manipulation is also an important aspect and effort sharing strategies have been proposed to improve the task performance [9].

From a control point of view, more versatility often means more complexity. A key element that emerges from previous research is that setting up dual-arm collaboration requires an appropriate task description. Initially introduced in [10], the cooperative task-space representation has been commonly adopted when dealing with dual-arm robots. Based on this approach, a task is defined by way of meaningful variables and can be kinematically solved using a dedicated Jacobian matrix [11]

Recently, the cooperative task-space representation has been used to perform physical human-robot collaboration [12], [13]. A cooperative control scheme based on an impedance law allows performing kinesthetic guiding operations. Adaptation of the stiffness along the trajectory grants the human co-worker with more accuracy during critical parts of the task.

In this paper, we propose to extend our dual-arm kinematic controller presented in [14] to make it suitable for physical human-robot collaboration. In particular, we focus on improving the quality of interaction during the collaborative carrying of an object.

First, assuming that wrench sensory information is available at the arms' wrist, we perform the appropriate transformations to estimate external and internal wrenches applied to the object. Using vision, we take into account the contact point at which the interaction occurs to produce a motion that better reflects the human intention. We also propose a method to estimate and cancel the object's gravity effects during comanipulation. This way, the external wrench generated by the object's mass does not interfere with human action.

The dual-arm controller implements an admittance control law that uses the task space measures to process the command. Depending on how the parameters are tuned, different control modes are available and can be selected independently for each task variable. For instance, force control may be combined with pose control to maintain an object with the two arms while moving it in the workspace. Compared to previous works on human-robot co-manipulation, the decoupled control on the task space variables allows to better manage the robot's behavior, to enhance the safety and the human feeling during the interaction.

We demonstrate the efficiency of our approach by performing experiments on a dual-arm robot. We evaluate the benefits of our strategy through a table-moving scenario carried out in collaboration with humans. Using virtual reality, we are able 


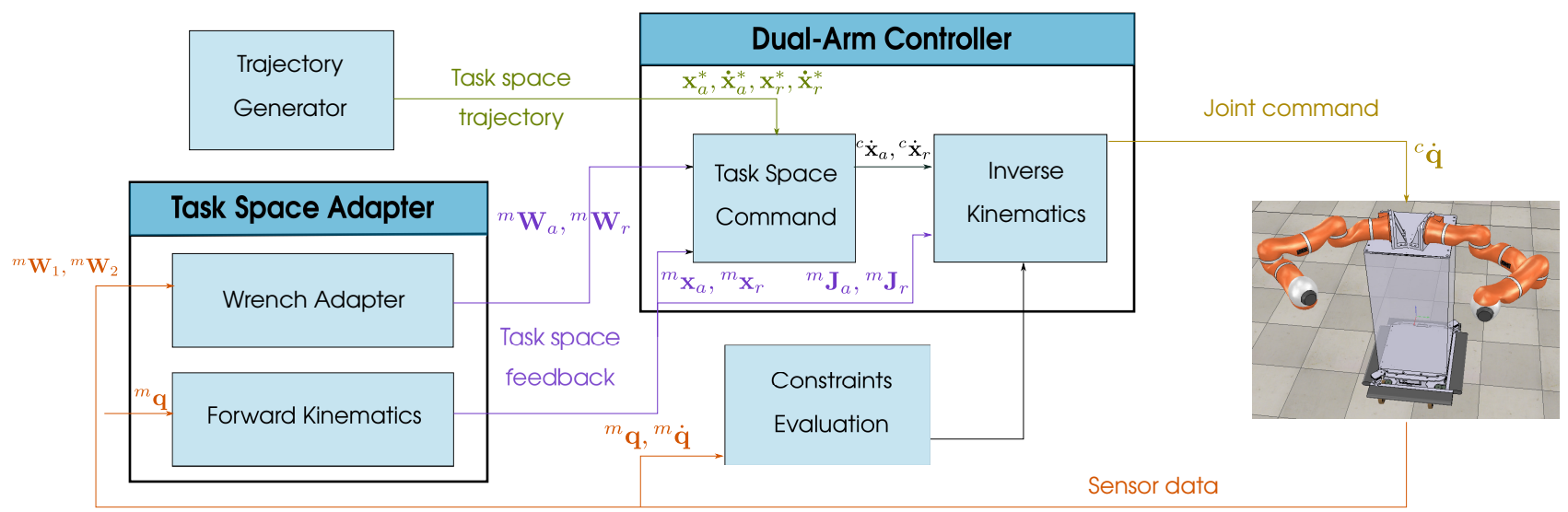

Fig. 1. Dual-arm kinematic control scheme. Based on the cooperative task representation [10], the absolute (subscript 'a') and relative (subscript 'r') tasks are controlled. The Task Space Adapter converts measurements (superscipt ' $m$ ') of joint positions ${ }^{m} \mathbf{q}$ and wrench ${ }^{m} \mathbf{W}_{1},{ }^{m} \mathbf{W}_{2}$ at the wrist of each arm into task space poses ${ }^{m} \mathbf{x}_{a},{ }^{m} \mathbf{x}_{r}$, wrenches ${ }^{m} \mathbf{W}_{a},{ }^{m} \mathbf{W}_{r}$ and extracts task space Jacobian matrices ${ }^{m} \mathbf{J}_{a},{ }^{m} \mathbf{J}_{r}$. The Dual-arm Controller then generates the command (superscipt 'c') at the task space level ${ }^{c} \dot{\mathbf{x}}_{a},{ }^{c} \dot{\mathbf{x}}_{r}$ from these feedback values and the desired (superscript '*') task space trajectory. Finally, the inverse kinematics provides the joint velocity command ${ }^{c} \dot{\mathbf{q}}$ sent to the robot.

to quantitatively compare the task performance when taking into account human intention or not. We point out that this consideration leads to smooth and effortless interaction with humans.

The paper is organized as follows. Section II gives an overview of our kinematic control scheme for dual-arm coordinated motions. Section III deals with wrench considerations for human-robot collaborative carrying. In Section IV we describe the task space admittance control law. We show how safe the manipulation of objects and interaction with the environment are possible. We present an experimental study in Section V, showing the benefits of our method through an object transportation scenario using computer vision and virtual reality. Section VI states the conclusion and future prospects.

\section{DUAL-ARM CONTROL SCHEME}

The framework for dual-arm collaborative manipulators proposed in [14] relies on the online closed-loop control scheme depicted in Fig. 1.

The proposed strategy uses wrench feedback. It allows us to manage internal constraints to safely manipulate an object without any risk of breakage but also to perceive external forces, essential information to perform humanrobot physical interaction. We adapt the cooperative task representation from [10] in a more robust and generic form to specify dual-arm operations in terms of absolute task (i.e. expressing the pose of any frame in the space with respect to a fixed world frame) and relative task (i.e. expressing the pose of one end-effector with respect to the other), as depicted in Fig. 2.

The Task Space Adapter block is in charge of converting feedback data coming from the robot into relevant information in the task space. Joint position feedback ${ }^{m} \mathbf{q}$ is interpreted by the Forward Kinematics process to evaluate the current state in the task space: it outputs the poses ${ }^{m} \mathbf{x}_{a},{ }^{m} \mathbf{x}_{r}$ and Jacobian matrices ${ }^{m} \mathbf{J}_{a},{ }^{m} \mathbf{J}_{r}$ linked with the absolute and relative tasks, respectively.
Wrench ${ }^{m} \mathbf{W}_{1},{ }^{m} \mathbf{W}_{2}$ (i.e., force and torques in operational space) measured at the tip of each end-effector are transformed by the Wrench Adapter block to have meaning in the task space through vectors ${ }^{m} \mathbf{W}_{a},{ }^{m} \mathbf{W}_{r}$.

Given a task specification (e.g. waypoints to reach), the Trajectory generator will provide desired poses $\mathbf{x}_{a}^{*}, \mathbf{x}_{r}^{*}$ and velocities $\dot{\mathbf{x}}_{a}^{*}, \dot{\mathbf{x}}_{r}^{*}$ to follow for the task space variables. The desired task space trajectory as well as the task space feedback are sent to the Dual-arm Controller which delivers the joint command to the robot. This operation is decomposed in two successive steps. First, the Task Space Command is generated based on the admittance control law that we will present in Section IV. The process can be tuned for each task variable in order to choose between position, force, or damping control modes.

Finally, the Inverse Kinematics block is processed. Based on a Hierarchical Quadratic Programming (HQP) architecture, relative and absolute tasks are treated in a prioritized way to ensure the proper manipulation of objects. Joint limits and collision avoidance are included as hard constraints to be fulfilled at all times.

Throughout the rest of the document, we assume the following:

- The absolute task expresses the end-effector pose of Arm 1 with respect to some fixed world frame $\mathcal{F}_{w}$ (superscript "w"). The corresponding homogeneous transformation matrix is $\mathbf{T}_{a}=\mathbf{T}_{1}^{w}$.

- Arm 1 is taken as reference in the relative task definition, leading to $\mathbf{T}_{r}=\mathbf{T}_{2}^{1}$.

- $\mathbf{W}_{1}, \mathbf{W}_{2}$ are measured with $\mathrm{F} / \mathrm{T}$ sensors mounted at the arms' wrist.

- Manipulated objects are firmly grasped by the two endeffectors.

\section{WRENCH FEEDBACK FOR COLLABORATIVE MANIPULATION}

Dual-arm mobile robots are particularly suited for transporting large and heavy objects. The versatility of such 


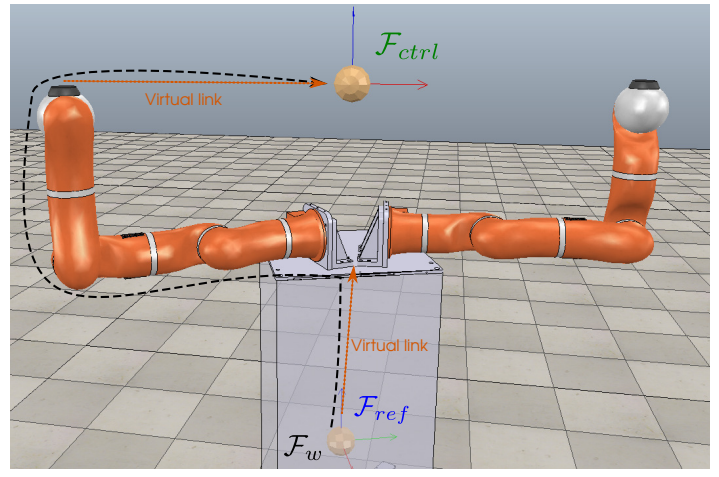

(a) The absolute task

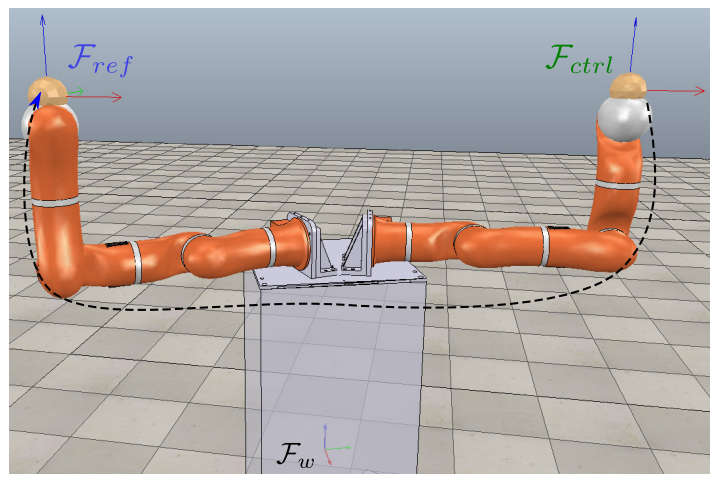

(b) The relative task

Fig. 2. Cooperative tasks representation. The absolute task expresses the pose of any arbitrary frame in the space $\left(\mathcal{F}_{c t r l}\right)$ with respect to a fixed world frame $\left(\mathcal{F}_{\text {ref }}\right)$. The kinematic chain associated with this task uses only one arm and virtual links for joining the frames of interest. The relative task expresses the pose of one end-effector $\left(\mathcal{F}_{c t r l}\right)$ with respect to the other $\left(\mathcal{F}_{\text {ref }}\right)$.

platforms can be better exploited by allowing human-robot cooperation. Notably, co-manipulation of a shared object is an interesting type of interaction in an industrial context. This can be used to facilitate the displacement of an object, by letting the human physically guide the robot towards the target location without lifting the weight of the object. Also, it can be applied to perform "teaching by demonstration" operations. This allows to quickly reconfigure the task of the robot without programming.

To properly exploit the wrench information, it has to be meaningful from the task space perspective. This conversion is made by the Wrench Adapter block depicted in Fig. 1. To improve the quality of the interaction during human-robot collaborative carrying, this block implements sequential processes to generate the cooperative task wrenches, as shown in Fig. 3.

First, wrench feedback coming from sensors is interpreted at the task frames. Then, the object weight is removed from the external wrench, as this should not be considered when interacting with the human. By knowing the interaction location, external moments are finally adapted to follow human intention.

Further, by performing damping control on selected absolute task variables (see Section IV), the robot will follow the motion driven by the human operator interacting with it.

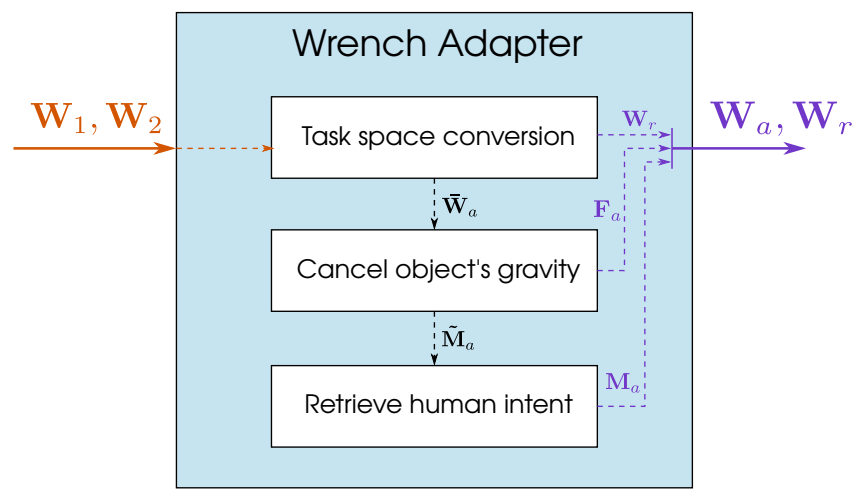

Fig. 3. The Wrench Adapter block is in charge of computing the cooperative task wrenches $\mathbf{W}_{a}, \mathbf{W}_{r}$ from wrench measurements at the end-effectors $\mathbf{W}_{1}, \mathbf{W}_{2}$. After conversion in the task space, sequential operations are performed to first remove object weight from the absolute task wrench $\overline{\mathbf{W}}_{a}$ and then retrieve human intention from the absolute task moment $\widehat{\mathbf{M}}_{a}$.

\section{A. Wrench evaluation in the task space}

We detail here the role of the Task space conversion block from Fig. 3.

Let us assume that an object is held by the two endeffectors, as depicted in Fig. 4. Forces $\mathbf{F}_{i}^{i}$ and moments $\mathbf{M}_{i}^{i}$ are perceived at the arms' wrists $(i=1,2)$ and gathered in the wrench vectors $\mathbf{W}_{1}^{1}$ and $\mathbf{W}_{2}^{2}$ that we write for simplicity $\mathbf{W}_{1}=\left[\begin{array}{ll}\mathbf{F}_{1} & \mathbf{M}_{1}\end{array}\right]^{T}$ and $\mathbf{W}_{2}=\left[\begin{array}{ll}\mathbf{F}_{2} & \mathbf{M}_{2}\end{array}\right]^{T}$. By using the principle of "virtual sticks" [15], the forces $\overline{\mathbf{F}}_{a}$ and moments $\overline{\mathbf{M}}_{a}$ exerted by the end-effectors at the absolute task frame $\mathcal{F}_{a}$ are defined as:

$$
\left\{\begin{aligned}
\overline{\mathbf{F}}_{a} & =\mathbf{F}_{1}^{w}+\mathbf{F}_{2}^{w}, \\
\overline{\mathbf{M}}_{a} & =\mathbf{M}_{1}^{w}+\mathbf{p}_{1, a}^{w} \times \mathbf{F}_{1}^{w}+\mathbf{M}_{2}^{w}+\mathbf{p}_{2, a}^{w} \times \mathbf{F}_{2}^{w},
\end{aligned}\right.
$$

where $\mathbf{p}_{1, a}^{w}, \mathbf{p}_{2, a}^{w}$, are vectors representing the "virtual sticks" joining at the absolute frame. The external wrench $\overline{\mathbf{W}}_{a}=\left[\begin{array}{ll}\overline{\mathbf{F}}_{a} & \overline{\mathbf{M}}_{a}\end{array}\right]^{T}$ corresponds to the resultant of all wrenches of the system applied to $\mathcal{F}_{a}$. It creates a linear mapping from the wrenches perceived at the end-effectors to the absolute task space.

The internal wrenches associated with the relative task do not affect the movements of the absolute frame, i.e. act in the null-space of $\overline{\mathbf{W}}_{a}$. There exists an infinite number of combinations of $\mathbf{W}_{1}$ and $\mathbf{W}_{2}$ that satisfy this condition. We keep the same definition as in [15] to compute the internal wrench vector $\overline{\mathbf{W}}_{r}=\left[\begin{array}{ll}\overline{\mathbf{F}}_{r} & \overline{\mathbf{M}}_{r}\end{array}\right]^{T}$ :

$$
\left\{\begin{aligned}
\mathbf{F}_{r} & =\frac{1}{2}\left(\mathbf{F}_{2}^{1}-\mathbf{F}_{1}^{1}\right), \\
\mathbf{M}_{r} & =\frac{1}{2}\left(\mathbf{M}_{2}^{1}-\mathbf{M}_{1}^{1}\right) .
\end{aligned}\right.
$$

The internal wrenches are neither affected by the object's gravity nor by the interaction with the human. Thus, the vector $\overline{\mathbf{W}}_{r}$ computed at this point corresponds to the final internal wrenches, such as $\overline{\mathbf{W}}_{r}=\mathbf{W}_{r}$. 


\section{B. Identification and cancellation of the objects' gravity effects}

When performing physical interaction with kinesthetic guidance through a commonly held object, its mass permanently applies external wrenches that should not be interpreted as a human action.

To overcome this undesired behavior, we propose a practical method to estimate and then cancel the effects of the payload.

Similarly to (1), the perceived external wrench in the object's frame $\mathcal{F}_{o}$ can be expressed with:

$$
\mathbf{W}_{o}^{w}=\left[\begin{array}{c}
\mathbf{F}_{1}^{w}+\mathbf{F}_{2}^{w} \\
\mathbf{M}_{1}^{w}+\mathbf{M}_{2}^{w}+\mathbf{F}_{1}^{w} \times \mathbf{p}_{1, o}^{w}+\mathbf{F}_{2}^{w} \times \mathbf{p}_{2, o}^{w}
\end{array}\right],
$$

where $\mathbf{p}_{1, o}^{w}$ and $\mathbf{p}_{2, o}^{w}$ denote the translation vectors going from each end-effector to the object's frame, expressed in the world's frame.

If we consider the manipulated object as a point mass under the sole influence of gravity (interaction being done at low speed/acceleration), this wrench can also be estimated as:

$$
\widehat{\mathbf{W}}_{o}^{w}=\left[\begin{array}{c}
m \mathbf{g} \\
\mathbf{R}_{o}^{w} \mathbf{c} \times m \mathbf{g}
\end{array}\right]
$$

where $m$ is the mass of the object, c its center of mass, expressed in the object's frame, and g the gravity vector in the world frame.

When considering practical object manipulation, $m$ and $\mathbf{c}$ are generally unknown or not well known. To estimate their parameters, one can collect measures while manipulating the object and use them in a non-linear optimization problem. Assuming that the grasping points are known, the problem can be formulated as follows:

$$
\begin{array}{ll}
\min _{\mathbf{Z}} & \left\|\mathbf{W}_{o}^{w}-\widehat{\mathbf{W}}_{o}^{w}\right\|_{2} \\
\text { s.t. } & m>0,
\end{array}
$$

where $\mathbf{z}$ is the vector of optimization variables:

$$
\mathbf{z}=\left[\begin{array}{c}
m \\
\mathbf{c}
\end{array}\right] \text {. }
$$

Once $\mathbf{z}$ that satisfies (5) has been found, it can be used to cancel objects' gravity effects. This operation is performed by the Cancel object's gravity block from Fig. 3 .

From the cooperative task perspective, the gravity effects of the manipulated object do not influence internal wrenches. They add however an additional component to the external wrenches, which should be subtracted to avoid interfering with the interaction.

Based on (1) and taking into account the objects' gravity effects, we can compute the external wrench vector $\tilde{\mathbf{W}}_{a}=$ $\left[\begin{array}{ll}\tilde{\mathbf{F}}_{a} & \tilde{\mathbf{M}}_{a}\end{array}\right]^{T}$ from which objects' gravity effects have been removed:

$$
\left\{\begin{aligned}
\tilde{\mathbf{F}}_{a} & =\mathbf{F}_{a}-m \mathbf{g} \\
\tilde{\mathbf{M}}_{a} & =\mathbf{M}_{a}-\mathbf{R}_{o}^{w} \mathbf{c} \times m \mathbf{g}
\end{aligned}\right.
$$

In (7), $\mathbf{R}_{o}^{w}$ is the rotation matrix expressing the center of mass $\mathbf{c}$ in the world frame.

The external force is not influenced by the location of the interaction during human-robot co-manipulation. The vector $\tilde{\mathbf{F}}_{a}$ computed at this point corresponds to the final external force, so that $\tilde{\mathbf{F}}_{a}=\mathbf{F}_{a}$.

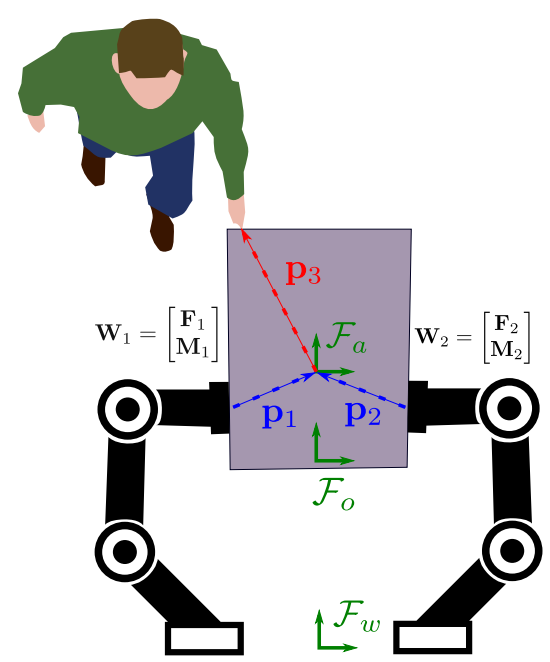

Fig. 4. Assuming a rigid grasp, wrenches applied at the absolute frame $\mathcal{F}_{a}$ are reconstructed considering the "virtual sticks" $\mathbf{p}_{1}$ and $\mathbf{p}_{2}$. Thereafter, external moments exerted by the human are retrieved from the "virtual stick" $\mathbf{p}_{3}$.

\section{Retrieve human intention}

During human-robot co-manipulation, the location on the object where the human exerts a force (called interaction point) has an incidence on what is perceived by the sensors. Indeed, considering the principle of levers, a linear force applied at some point of the object may create a moment at the grasping points. The bigger the object is, the more the perception is disturbed.

In [16], Dumora et al. proposed a statistical model to identify the human intention during shared human-robot collaborative task based on haptic measures only.

In this work, we assume that the interaction point is known (e.g. using visual monitoring). Considering that the weight of the object has been priorly canceled, it is possible to infer the human intention by removing undesired components of the external moment vector $\tilde{\mathbf{M}}_{a}$ coming from the lever action. In contrast with [16], this methods gives an accurate result.

Reusing the principle of "virtual sticks", one can easily retrieve the external moments $\mathbf{M}_{a}$ applied at the interaction point from the following equation:

$$
\mathbf{M}_{a}=\tilde{\mathbf{M}}_{a}+\mathbf{F}_{a} \times \mathbf{p}_{3, a}^{w}
$$

where $\mathbf{p}_{3}^{w}$ is the translation vectors going from the absolute task frame to the interaction point, as depicted in Fig. 4. Note that the computed wrench is still associated with the absolute task control point defined by $\mathcal{F}_{a}$. 


\section{TASK SPACE ADMITTANCE CONTROL}

In an open-loop system, the task space command would be directly issued from the trajectory generator and converted into joint commands without worrying about the evolution of the robot and the environment. However, in an unstructured workspace in which physical interaction with human operators may occur, it is necessary to close the feedback loop. As presented in the previous section, the feedback data coming from the different sensors are interpreted in the task space. Here, the feedforward trajectory is combined with this information to adapt the command.

To let the robot interact with the environment, let us consider virtual spring-damper mechanisms attached to each control point, as illustrated in Fig. 5.

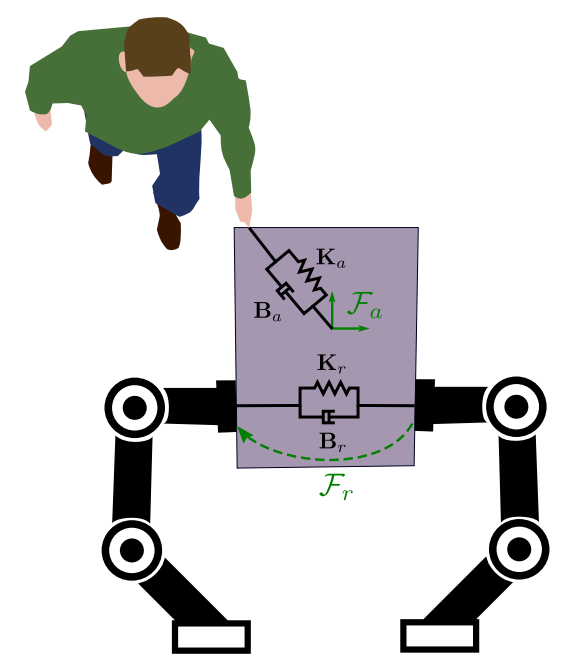

Fig. 5. Representation of the virtual spring-damper systems attached to the relative and absolute task control points.

The task space control law is deduced from the virtual spring-damper system dynamic equation:

$$
\left\{\begin{array}{l}
\mathbf{W}_{a}=\mathbf{K}_{a} \Delta \mathbf{x}_{a}+\mathbf{B}_{a} \Delta \dot{\mathbf{x}}_{a} \\
\mathbf{W}_{r}=\mathbf{K}_{r} \Delta \mathbf{x}_{r}+\mathbf{B}_{r} \Delta \dot{\mathbf{x}}_{r}
\end{array}\right.
$$

In the remainder of this section, without loss of generality, we will treat the general case of admittance control applied to a single task space DOF (without distinguishing between the absolute and relative task), as there is no coupling between variables. Every term is now expressed as a scalar value. It gives:

$$
W=K \Delta x+B \Delta \dot{x},
$$

with $\Delta x=x-x^{*}, \Delta \dot{x}=\dot{x}-\dot{x}^{*}$ being the error between the current and desired (superscript $*$ ) task pose and velocity, respectively. This equation relates the wrench $W$ applied by the spring-damper system on the control point and the pose $x$ of this control point by means of a proportional-derivative controller. The stiffness $K$ and the damping $B$ are the gain values for this DOF.

During dual-arm grasping of an object, it is often preferable to regulate the internal stress to prevent any damage to the object. To make this possible, we include the desired wrench value term $W^{*}$ in the previous equation:

$$
\Delta W=K \Delta x+B \Delta \dot{x},
$$

with $\Delta W=W-W^{*}$ expressing the error on the wrench regulation.

To realize admittance control, (11) can be rewritten in the following form:

$$
\dot{x}=\dot{x}^{*}+B^{-1}(\Delta W-K \Delta x),
$$

\section{EXPERIMENTAL STUDY}

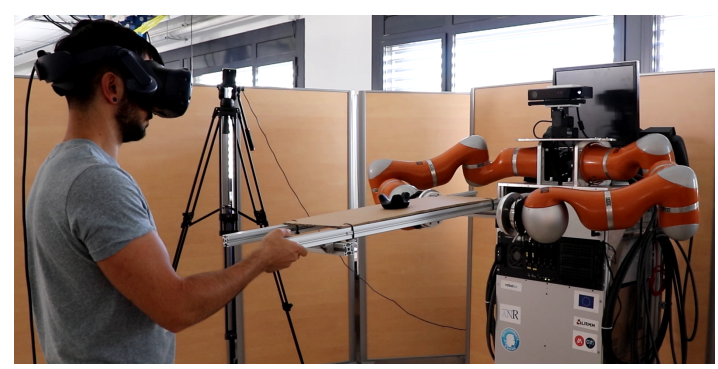

(a) Experimental setup
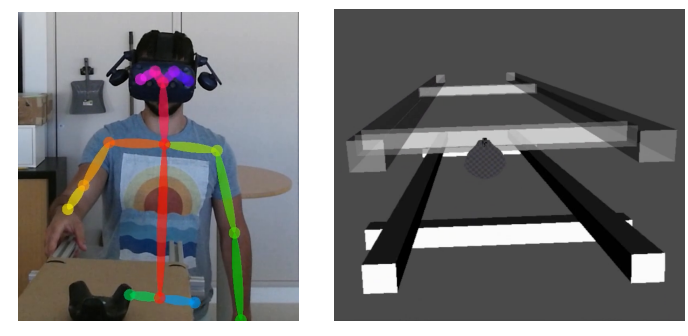

(b) Model of the user (c) Virtual objects seen in the skeleton. HMD.

Fig. 6. Human-robot co-manipulation of a large object using damping control. The interaction point is detected by the computer vision system. The current and target location of the object are displayed in the Head Mounted Display.

\section{A. Scenario}

The proposed approach has been evaluated through an experimental study conducted on the dual-arm cobot BAZAR [17]. To assess human intention consideration during cooperative manipulation, we set up an original simulated application scenario: selected participants were asked to achieve interactive tasks with the robot in the form of a table-moving scenario. The dual-arm robot is in charge of maintaining the table from one side by regulating internal efforts while the human operator grasps it on the opposite side with only one hand. The objective is to move the object, with damping control, from an initial pose in the space to a final one. The human motion should remain as natural as possible (taking the shortest distance to reach the goal and applying reasonable efforts).

During the experiments, participants wear a head-mounted display (HMD) in which the carried table is displayed both at the current (solid) and goal (transparency) location, as 
depicted in Fig. 6(c). The participants' task is to bring the solid object on top of the transparent one. The current pose is tracked online and its visualization in the virtual environment is updated continuously. This way, the user determines the motion needed to complete the task. Participants randomly execute the operation with/without the interaction point consideration without knowing it. A screenshot of the experiment is given in Fig. 6(a).

Three different operations have been elaborated for this study. In any case, the relative task is kept in a constant position on every component except for the $\mathrm{z}$ translational axis for which the force is regulated (to maintain the object properly). A damping term is added to the position control to give some compliance to the system. From a constant initial pose, a transform is applied to the absolute task for the three different cases:

1) trany+: translation of $25 \mathrm{~cm}$ along y axis. The user can move the object in the horizontal plane (in translation and rotation).

2) tranz+: translation of $25 \mathrm{~cm}$ along $\mathrm{z}$ axis. The user can move the object in the vertical plane in translation and rotate around y axis.

3) rotz+: rotation of $0.5 \mathrm{rad}$ around $\mathrm{z}$ axis. The user can move the object in the horizontal plane (in translation and rotation).

Video of the experiments is available at http://bit. do/e3mxM.

\section{B. Setup}

The BAZAR robot is equipped with two 7-DOF Kuka LWR4 arms. All experiments are performed on a computer with an Intel(R) Xeon(R) E5-2620 v3 CPU running Linux with the PREEMPT-RT patch. Our approach has been implemented in $\mathrm{C}++$ using the RKCL framework. The Fast Research Interface Library (FRI) ${ }^{1}$ is used to communicate with the Kuka arms, and the controller sample time was set to $T=5 \mathrm{~ms}$.

We tune the gains depending on the control mode: for compliant pose control, we set $B=150, K=250$ for forces and $B=25, K=40$ for torques; same gains are used for damping controlled variables but the stiffness term is removed $(K=0)$; for force-controlled variables, the stiffness gain is also $K=0$ while the damping term is $B=1000$ for forces and $B=500$ for torques.

We use computer vision to estimate online the position of the interaction point. The algorithm is based on the OpenPose library [18] which extracts the set of 2D points composing the skeleton of the persons present in a given color image. By using a Microsoft Kinect V2 RGB-D camera, we can reproject these $2 \mathrm{D}$ points in $3 \mathrm{D}$ using the depth information provided by the sensor, as shown in Fig. 6(b). We use the right-hand wrist as the interaction point since OpenPose doesn't provide a point for the hand itself. This allows us to position the point of interaction with an accuracy of a few centimeters ${ }^{2}$, which is sufficient for our application. In

\footnotetext{
${ }^{1}$ https://cs.stanford.edu/people/tkr/fri/html/

${ }^{2}$ Accuracy decreases with distance due to depth inacuraries
}

the case of occlusions, the last interaction point, expressed in the absolute frame, is kept. This hypothesis holds as long as the operator does not reposition his/her hand while the occlusion occurs. By using an NVIDIA GTX 1080 Ti GPU, we achieve around 7 estimations per second.

As mentioned in $\mathrm{V}$-A, in order to get reproducible and accurate results to validate the proposed approach, we set up a virtual reality (VR) system to instruct the operator on the task to achieve. This system is composed of an HTC Vive Pro HMD, an HTC Vive tracker attached to the transported object and two fixed Steam VR base stations. This VR system was only used for validation purposes and is not required in normal operations.

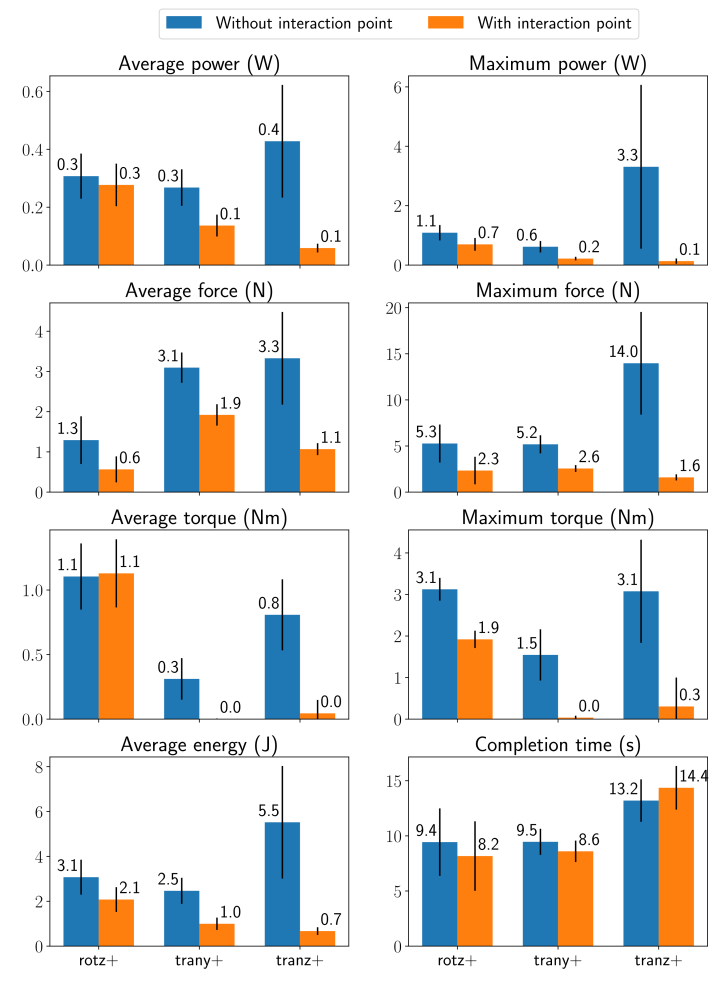

Fig. 7. Statistical data comparing several metrics for the three different tasks when the interaction point is taken into account (orange) or not (blue). Results showcase the benefits of our method since less effort is required by the participants to achieve the tasks.

\section{Results}

Ten participants performed a total of 15 operations (five for each case, selected in random order). Several criteria have been evaluated and statistical results are given in Fig. 7. Instantaneous power $P$ has been obtained using the relation $P=|\overrightarrow{\mathbf{W}} \cdot \overrightarrow{\mathbf{x}}|$ and the average energy $\bar{E}$ expended during a time interval $\Delta t$ arises from $\bar{E}=\bar{P} * \Delta t ; \bar{P}$ being the average power supplied during the same time interval.

A general observation is that the interaction point consideration greatly improves task performances. Indeed, the various metrics indicate that all the tasks can be completed effortlessly when the interaction point is known (orange bars) while it is much more difficult otherwise (blue bars). 
A closer look reveals that the differences are more noticeable for the translation tasks for which the necessary energy is between 2.5 (for the trany+ task) and 7.9 (for the tranz+ task) times higher when the interaction point is unknown. By contrast, the gap is smaller for the rotation task with a ratio of 1.5 for the average energy. The power consumption is even equivalent in this case with around $0.3 \mathrm{~W}$ on average. Referring to (8), this can be explained by the fact that the interaction point has an influence on the final wrench only in the presence of translational forces. In practice, it is almost impossible to apply perfect torque on the object without exerting any residual translation. Thus, the surplus energy supplied to complete the rotx task without interaction point may result from the greater effort generated to compensate for the translational error $(1.3 \mathrm{~N}$ against $0.6 \mathrm{~N})$, as the motion is less intuitive in this case.

The task tranz+ is the one that shows the strongest contrast when comparing the metrics. In particular, the maximum values are extremely higher when the interaction point is not taken into account. This is also the task in which the data are the most irregular (highlighted by standard deviation significant values). It seems that, depending on the participant, the unwanted torque generated when the interaction point is omitted creates some orientation error that is not trivial to compensate. It would appear that a task on the horizontal plane is easier to get under control when some error occurs.

The completion time is not significantly influenced by the method that is used, and is more dependent on the person realizing the task.

\section{CONCLUSION}

This paper addressed the problem of object comanipulation with a dual-arm robot, which is a very attractive kind of human-robot collaboration for industrial purposes. Indeed, combining the skills of humans (intelligence, versatility) with the powerful capabilities of robots (precision, repeatability) has a tremendous potential to increase the productivity of the manufacturing industry.

However, physical interactions between robots and humans have to be executed under adequate conditions to be natural and efficient. For that purpose, we extended our previous works on dual-arm kinematic control strategy to include new considerations which aim at improving the quality of cooperative tasks. Notably, we developed an online method for estimating gravity parameters of the manipulated object. Then, by applying some treatments on the wrench feedback data during collaborative operations, we are able to compensate the load exerted on the robotic arms to keep only external efforts applied by the human.

Furthermore, the human's contact location with the object is crucial information allowing to retrieve his intention from wrench measured at the arms' wrist. An experimental study has been elaborated to simulate a table-moving scenario using a virtual reality system and computer vision to detect the participant's hands during co-manipulation. The results clearly indicate that considering the interaction point is beneficial for co-manipulation tasks as it is more intuitive and thus requires less energy to be completed.

Future works will be dedicated to validate the approach with different objects of various shapes and on more complex tasks.

\section{ACKNOWLEDGMENT}

This work has received funding from the EU H2020 research and innovation programme as part of the project VERSATILE (grant agreement No 731330).

\section{REFERENCES}

[1] C. Smith, Y. Karayiannidis, L. Nalpantidis, X. Gratal, P. Qi, D. V. Dimarogonas, and D. Kragic, "Dual arm manipulationa survey," Robotics and Autonomous Systems, vol. 60, no. 10, pp. 1340-1353, 2012.

[2] J. Felip and A. Morales, "A solution for the cap unscrewing task with a dual arm sensor-based system," in IEEE-RAS Int. Conf. on Humanoid Robots, 2014.

[3] M. P. Polverini, A. M. Zanchettin, F. Incocciati, and P. Rocco, "Robust constraint-based robot control for bimanual cap rotation," in IEEE/RSJ Int. Conf. on Robots and Intelligent Systems, 2017, pp. 4785-4790.

[4] Z. Xian, P. Lertkultanon, and Q.-C. Pham, "Closed-chain manipulation of large objects by multi-arm robotic systems," IEEE Robotics and Automation Letters, vol. 2, no. 4, pp. 1832-1839, 2017.

[5] A. Cherubini, R. Passama, A. Crosnier, A. Lasnier, and P. Fraisse, "Collaborative manufacturing with physical human-robot interaction," Robotics and Computer-Integrated Manufacturing, vol. 40, pp. 1-13, 2016.

[6] K. Kosuge, H. Yoshida, D. Taguchi, T. Fukuda, K. Hariki, K. Kanitani, and M. Sakai, "Robot-human collaboration for new robotic applications," in Proceedings of IECON'94-20th Annual Conf. of IEEE Industrial Electronics, vol. 2. IEEE, 1994, pp. 713-718.

[7] K. Kosuge, M. Sato, and N. Kazamura, "Mobile robot helper," in IEEE Int. Conf. on Robotics and Automation, vol. 1, 2000, pp. 583-588.

[8] T. Takubo, H. Arai, Y. Hayashibara, and K. Tanie, "Human-robot cooperative manipulation using a virtual nonholonomic constraint," The Int. Journal of Robotics Research, vol. 21, no. 5-6, pp. 541-553, 2002.

[9] M. Lawitzky, A. Mörtl, and S. Hirche, "Load sharing in human-robot cooperative manipulation," in IEEE Int. Symposium on Robot and Human Interactive Communication, 2010, pp. 185-191.

[10] P. Chiacchio, S. Chiaverini, and B. Siciliano, "Direct and inverse kinematics for coordinated motion tasks of a two-manipulator system," Journal of dynamic systems, measurement, and control, vol. 118, no. 4, pp. 691-697, 1996.

[11] R. S. Jamisola and R. G. Roberts, "A more compact expression of relative jacobian based on individual manipulator jacobians," Robotics and Autonomous Systems, vol. 63, no. P1, pp. 158-164, 2015.

[12] B. Nemec, N. Likar, A. Gams, and A. Ude, "Bimanual human robot cooperation with adaptive stiffness control," in IEEE-RAS Int. Conf. on Humanoid Robots, 2016, pp. 607-613.

[13] — - "Adaptive human robot cooperation scheme for bimanual robots," in Advances in Robot Kinematics. Springer, 2018, pp. 371380 .

[14] S. Tarbouriech, B. Navarro, P. Fraisse, A. Crosnier, A. Cherubini, and D. Sallé, "Mobile dual-arm collaborative framework," Robotics and Autonomous Systems (under review), 2019. [Online]. Available: http://bit.do/e3qMx

[15] M. Uchiyama and P. Dauchez, "A symmetric hybrid position/force control scheme for the coordination of two robots," in IEEE/RSJ Int. Conf. on Robots and Intelligent Systems, 1988, pp. 350-356.

[16] J. Dumora, F. Geffard, C. Bidard, T. Brouillet, and P. Fraisse, "Experimental study on haptic communication of a human in a shared human-robot collaborative task," in IEEE/RSJ Int. Conf. on Robots and Intelligent Systems, 2012, pp. 5137-5144.

[17] A. Cherubini, R. Passama, B. Navarro, M. Sorour, A. Khelloufi, O. Mazhar, S. Tarbouriech, J. Zhu, O. Tempier, A. Crosnier, et al., "A collaborative robot for the factory of the future: Bazar," The Int. Journal of Advanced Manufacturing Technology, pp. 1-17, 2019.

[18] Z. Cao, G. Hidalgo, T. Simon, S.-E. Wei, and Y. Sheikh, "OpenPose: realtime multi-person 2D pose estimation using Part Affinity Fields," in arXiv preprint arXiv:1812.08008, 2018. 